\title{
Heating Effects Induced in a Finite Silver Selenide Slab by a Modelled Laser Internal Source Using the Hyperbolic Heat Conduction (HHCE) Model in Dimensionless Domain
}

\author{
Mohamed Abdelhady Kamel El-Adawi ${ }^{1, ~ *, ~ S a f a a ~ A b d e l f a t t a h ~ S h a l a b y ~}{ }^{1}$, Hoda Saad Al-Fanakh ${ }^{2}$ \\ ${ }^{1}$ Physics Department, Faculty of Education, Ain Shams University, Cairo, Egypt \\ ${ }^{2}$ Physics Department, Faculty of Science for Girls, Dammam University, Dammam, Saudia Arabia
}

Email address:

adawish1@hotmail.com(M. A. K. El-Adawi)

${ }^{*}$ Corresponding author

\section{To cite this article:}

Mohamed Abdelhady Kamel El-Adawi, Safaa Abdelfattah Shalaby, Hoda Saad Al-Fanakh. Heating Effects Induced in a Finite Silver Selenide Slab by a Modelled Laser Internal Source Using the Hyperbolic Heat Conduction (HHCE) Model in Dimensionless Domain. International Journal of Applied Mathematics and Theoretical Physics. Vol. 5, No. 1, 2019, pp. 32-37. doi: 10.11648/j.ijamtp.20190501.14

Received: March 11, 2019; Accepted: April 16, 2019; Published: May 20, 2019

\begin{abstract}
Lasers of high power densities are useful for a variety of material processing techniques. Laser heating of a finite homogeneous Silver Selenide slab is studied according to the hyperbolic heat conduction model. Laplace Integral transform technique is used to get the solution. This material suffers phase transition from semiconductor to metallic phase at $403 \mathrm{~K}$. It has vital technological applications. The obtained temperature field makes it possible to determine the time required to initiate phase transition or melting. The functional dependence of the obtained functions is revealed. Different laser power densities are considered as illustrative examples.
\end{abstract}

Keywords: Laser Heating, Internal Heating Source, (HHCE) Heating Model, Phase Transition in Silver Selenide Slab, Laplace Integral Transform in Heating Problems

\section{Introduction}

There is an increasing interest to study laser interaction with matter.

Laser sources of high power density up to $10^{12} \mathrm{~W} / \mathrm{m}^{2}$ or more and that of moderate power densities have vital applications in different fields especially for material processing techniques such as spot, welding, surface annealing, drilling of metals, laser cutting of metals, laser shock hardening, laser glazing, measuring the physical properties of thin films, local diffusion and alloying to form (p-n) junctions, optical recording [1-6].

In many of such studies and applications it is particularly important to find theoretically the temperature field within the irradiated target. This makes it possible to determine the critical time required to initiate phase transition and also to determine the critical time required to initiate melting. For multi-phase problems, it is important to find the rate of melting or the rate of evaporation of the considered target. The study of laser damage in some of the irradiated materials is important to avoid damage as in the case of laser mirrors [7].

There are two trends in such a study. The first trend is the Fourier model that assumes infinite speed of heat transport, i.e. a local thermal disturbance is instantaneously felt at each point of the irradiated material. This is formulated through the parabolic heat conduction equation (PHCE). The second trend which is more realistic one is based on the Cattaneo flux law. This leads to the hyperbolic heat conduction equation (HHCE).

Accoding to (HHCE) heat is suggested to propagate with finite velocity. This is more suitable when extremely short laser pulses or laser of very high frequencies are considered [8].

The present trial deals with heating a finite silver selenide $\left(\mathrm{Ag}_{2} \mathrm{Se}\right)$ slab.

The considered material $\left(\mathrm{Ag}_{2} \mathrm{Se}\right)$ has applications in different technological fields. It acts as a thermoelectric power generator material [9]. It has an important role in the field of switching devices $[10,11]$. Such a material 
undergoes through phase transition. It has two phases [10]: $\beta$ phase with orthorhombic structure (below $400^{\circ} \mathrm{K}$ ) and $\alpha-$ phase (for temperature level greater than $400^{\circ} \mathrm{K}$ ) with bodycentered-cubic (bcc). Morover, the considered material shows semiconductor properties up to $403^{\circ} \mathrm{K}$, and then behaves as a metal [10].

The objective of the present study is to find analytically the temperature field induced within the irradiated target $\left(\mathrm{Ag}_{2} \mathrm{Se}\right)$ by an internal laser source with volume capacity "g" $\mathrm{W} / \mathrm{m}^{3}$.

Hyperbolic heat conduction equation ((HHCE) in dimensionless domain is solved. Laplace integral transform technique makes it possible to get the solution. The obtained expression for the temperature field enables to find the variation of the front surface temperature with the exposure time. From which one can determine the critical time required to initiate phase transition $\left(\mathrm{t}_{\mathrm{ph}}\right)$ and that required to initiate melting " $\mathrm{t}_{\mathrm{m}}$ ". Thus the functional dependences of such functions are clarified. Computations dealing with some illustrative examples considering different operating conductions are given.

\section{Mathematical Formulation}

According to the hyperbolic model of heat conduction, the modified Fourier equation (Cattaneo equation) has the form [8]:

$$
t_{k} \frac{\partial q(x, t)}{\partial t}+q(x, t)=-\lambda \nabla T(x, t)
$$

The energy conservation (continuity) equation is written in the form:

$$
\rho c_{p} \frac{\partial T}{\partial t}=-\nabla \cdot q+g
$$

Equation (1) is incorporated with equation (2) to give the following hyperbolic equation of heat conduction (HHCE):

$$
\begin{gathered}
t_{k} \frac{\partial^{2} T}{\partial t^{2}}+\frac{\partial T}{\partial t}=\alpha \nabla^{2} T+\frac{1}{\rho c_{p}}\left(t_{k} \frac{\partial g}{\partial t}+g\right), \\
0 \leq x \leq d \quad, t \geq 0
\end{gathered}
$$

In equation (1), (2), $t_{k}=\frac{\alpha}{w^{2}} \quad, \sec$ is the thermal relaxation time characterizing a time scale of the flux relaxation process, $\alpha=\frac{\lambda}{\rho c_{p}}, \frac{m^{2}}{\mathrm{sec}}$, is the thermal diffusivity written in terms of the heat thermal conductivity $\lambda$ and heat capacity per unit volume $\left(\rho c_{p}\right)$. and $W, m / s e c$ is the speed of propagation of the thermal wave in the target material.

For a material that absorbs laser energy internally, the internal energy source " $\mathrm{g}$ " is modeled as:

$$
g(x, t)=q_{0}(0, t)(1-R) \mu \exp (-\mu x)
$$

$q_{0}(0, \mathrm{t}), \mathrm{W} / \mathrm{m}^{2}$ is the external laser irradiance incident on the font surface of the target.

$R$, is the front surface reflectance of the slab, and $\mu$ is the absorption attenuation coefficient.

Equation (4) assumes no spatial variations of the incident laser intensity in the plane perpendicular to the incident laser beam direction and no heat transport in the direction perpendicular to the direction of the incident beam $[2,5,13]$. Thus one dimensional case is considered.

For such a case, the diameter of the laser beam is also assumed to be large compared to the thickness of the slab.

Equation (3) is subjected to the following initial and boundary condition:

$$
\begin{gathered}
\text { At } t=0 \quad T(x, 0)=0 \\
\text { At } x=\left.d \quad \frac{\partial T}{\partial x}\right|_{x=d}=0 \\
\text { At } x=\left.0 \quad \frac{\partial T}{\partial x}\right|_{x=0}=-\frac{1}{\lambda}\left[q(0, t)+t_{k} \frac{\partial q}{\partial t}(0, t)\right]
\end{gathered}
$$

Condition (6) indicates that the slab in thermally insulated at the rear surface.

To convert the hyperbolic equation (3) to be in dimensionless coordinates, let us consider the following dimensionless variables defined as $[8,14]$ :

$$
\begin{gathered}
X=\frac{W x}{2 \alpha} \\
Y=\frac{W Y}{2 \alpha} \\
Z=\frac{W z}{2 \alpha} \\
\theta=\frac{\left(T-T_{0}\right)}{\left(T_{m}-T_{0}\right)} \\
\varphi=\frac{q(0, t)(1-R)}{W \rho c_{p}\left(T_{m}-T_{0}\right)} \\
\tau=\frac{t}{2 t_{k}} \\
\psi=\frac{g t_{k}}{\rho c_{p}\left(T_{m}-T_{0}\right)}
\end{gathered}
$$

$T_{0},{ }^{\circ} \mathrm{K}$ is the ambient temperature, $T_{m},{ }^{\circ} \mathrm{K}$ is the melting temperature of the target material.

One obtains Cattaneo (modified Fourier) equation in the form:

$$
\frac{\partial \varphi}{\partial \tau}+2 \varphi=-\nabla \theta
$$


Moreover, the following two equations are obtained:

The energy conservation equation:

$$
\frac{\partial \varphi}{\partial \tau}+2 \varphi=-\nabla \theta+2 \psi
$$

The hyperbolic heat conduction equation:

$$
\frac{\partial^{2} \theta}{\partial \tau^{2}}+2 \frac{\partial \theta}{\partial \tau}=\frac{\partial^{2} \theta}{\partial X^{2}}+2 \frac{\partial \psi}{\partial \tau}+4 \psi
$$

The dimensionless heat source capacity is given in the form [8]

$$
\psi(X, \tau)=\psi_{0} \eta(\tau) \exp (-\beta X)
$$

Where,

$$
\eta(\tau)=\frac{q_{0}\left(2 t_{k} \tau\right)}{q_{r}}, \quad q_{0}=\eta q_{r}
$$

And

$$
\begin{gathered}
\psi_{0}=\frac{g_{r}(1-R) \mu t_{k}}{\rho c_{p}\left(T_{m}-T_{0}\right)} \\
\beta=2 W t_{k} \mu
\end{gathered}
$$

$q_{r}, \mathrm{~W} / \mathrm{m}^{2}$ arbitrary reference lase intensity, [8]

$\eta$, Dimensionless laser energy absorbed in the medium $\beta$, Dimensionless attenuation coefficient

Introducing the expression of $\psi(X, \tau)$ equation ( 18$)$ into equation (17) one gets the (HHCE) in the form [8]:

$$
\frac{\partial^{2} \theta}{\partial \tau^{2}}+2 \frac{\partial \theta}{\partial \tau}=\frac{\partial^{2} \theta}{\partial X^{2}}+2 \psi_{0}\left(\frac{\partial \eta}{\partial \tau}+2 \eta\right) \exp (-\beta X)
$$

Equation (22) is subjected to the following initial and boundary conditions in the dimensionless scales as:

$$
\begin{aligned}
& \text { At } \tau=0 \quad \theta(X, 0)=0 \\
& \text { At } \quad X=\frac{W d}{2 \alpha}=\left.X_{d} \quad \frac{\partial \theta}{\partial X}\right|_{X=X_{d}}=0 \\
& \text { At } \quad X=0 \quad \frac{\partial \theta}{\partial X}=-\left[2 \varphi(0, \tau)+\frac{\partial \varphi}{\partial \tau}(0, \tau)\right] \\
& \bar{\theta}(X, S)=\frac{-4 \beta \psi_{0} \eta e^{-\sqrt{S(S+2)} X}}{S \sqrt{S(S+2)}\left[S(S+2)-\beta^{2}\right]}
\end{aligned}
$$

Comparing the order of magnitude of different terms in equation (34) reveals that the first term can be neglected, thus:

$$
\bar{\theta}(X, S)=\frac{2 \varphi e^{-\sqrt{S(S+2)} X}}{\sqrt{S(S+2)}}+\frac{4 \psi_{0} \eta e^{-\beta X}}{S\left[S(S+2)-\beta^{2}\right]}
$$

Let us solve equation (22) for the case when $\mathrm{q}_{\mathrm{o}}=$ constant, modeled as a unit step function.

Taking Laplace integral transform for equation (22) with respect to time $\tau$ one gets:

$$
\frac{\partial^{2}}{\partial X^{2}} \bar{\theta}(X, S)-S(S+2) \bar{\theta}(X, S)=-\frac{4 \psi_{0} \eta e^{-\beta X}}{S}
$$

Let

$$
\bar{\theta}(X, S)=\bar{\theta}_{H}(X, S)+\bar{\theta}_{p}(X, S)
$$

Let us find the solution of the homogeneous part $\bar{\theta}_{H}$ :

$$
\frac{\partial^{2}}{\partial X^{2}} \bar{\theta}_{H}(X, S)-S(S+2) \bar{\theta}_{H}(X, S)=0
$$

The solution is obtained in the form:

$$
\bar{\theta}_{H}(X, S)=A e^{\sqrt{S(S+2)} X}+B e^{-\sqrt{S(S+2)} X}
$$

To get a finite solution at infinity we put $A=0$, thus:

$$
\bar{\theta}_{H}(X, S)=B e^{-\sqrt{S(S+2)} X}
$$

To find a particular solution $\bar{\theta}_{p}(X, S)$ for equation (26) let us use the inverse differential operator technique [15], this gives:

$$
\bar{\theta}_{p}(X, S)=-\frac{4 \psi_{0} \eta e^{-\beta X}}{S\left[S(S+2)-\beta^{2}\right]}
$$

Equation (30) and (31) lead to the following solution of equation (26):

$$
\bar{\theta}(X, S)=B e^{-\sqrt{S(S+2)} X}+\frac{4 \psi_{0} \eta e^{-\beta X}}{S\left[S(S+2)-\beta^{2}\right]}
$$

Let us apply the boundary condition at $X=0$ (equation (25) to equation (32) to get the factor $B$ in the form:

$$
B=-\frac{4 \beta \psi_{0} \eta}{S \sqrt{S(S+2)}\left[S(S+2)-\beta^{2}\right]}+\frac{2 \varphi}{\sqrt{S(S+2)}}
$$

Substituting equation (33) into equation (32) one gets the solution in the form:

$+\frac{2 \varphi e^{-\sqrt{S(S+2)} X}}{\sqrt{S(S+2)}}+\frac{4 \psi_{0} \eta e^{-\beta X}}{S\left[S(S+2)-\beta^{2}\right]}$

Consider the inverse Laplace transform $[16,17,18]$ :

$L^{-1} \frac{e^{-k \sqrt{S(S+a)}}}{\sqrt{S(S+a)}}=e^{-\frac{1}{2} a t} I_{\circ}\left[\frac{1}{2} a \sqrt{t^{2}-k^{2}}\right] u(t-k), K \geq 0$ 
Where the modified Bessel function $I_{\circ}$ is defined as:

$$
I_{\circ}(x)=\sum_{n=1}^{\infty} \frac{\left(\frac{x}{2}\right)^{2 n}}{n !} \Gamma(n+1)
$$

The step function $u(t-k)$ is defined as:

$$
\begin{aligned}
& u(t-K)= \begin{cases}0 & \text { at } \quad(t-K)<0 \\
\frac{1}{2} & \text { at }(t-K)=0 \\
1 & \text { at }(t-K)>0\end{cases} \\
& L^{-1} \frac{1}{\left[(S-b)^{2}-a^{2}\right]}=e^{b t} \frac{\sinh a t}{a} \\
& L^{-1}\left\{\frac{4 \psi_{0} \eta e^{-\beta X}}{S\left[S(S+2)-\beta^{2}\right]}\right\}=4 \psi_{0} \eta e^{-\beta X}\left[\int_{0}^{\tau} e^{-\tau} \frac{\sinh \sqrt{\left(\beta^{2}+1\right)} \tau}{\sqrt{\left(\beta^{2}+1\right)}} d \tau\right] \\
& =4 \psi_{0} \eta e^{-\beta X}\left[\int_{0}^{\tau} \frac{\left[e^{+\left[\sqrt{\beta^{2}+1}-1\right] \tau}-e^{-\left[\sqrt{\beta^{2}+1}+1\right] \tau}\right]}{2 \sqrt{\left(\beta^{2}+1\right)}} d \tau\right] \\
& =4 \psi_{0} \eta e^{-\beta X} \frac{1}{2 \sqrt{\left(\beta^{2}+1\right)}}\left[\frac{e^{+\left[\sqrt{\beta^{2}+1}-1\right] \tau}-1}{\sqrt{\beta^{2}+1}-1}+\frac{e^{-\left[\sqrt{\beta^{2}+1}+1\right] \tau}-1}{\sqrt{\beta^{2}+1}+1}\right]
\end{aligned}
$$
one obtains:

For the second term let us consider:

Thus for the first term of equation (35), put $k=X, a=2$

$$
L^{-1}\left\{\frac{2 \varphi e^{-\sqrt{S(S+2)} X}}{\sqrt{S(S+2)}}\right\}=2 \varphi e^{-\tau} \sum_{n=1}^{\infty} \frac{\left(\frac{X}{2}\right)^{2 n}}{n !} \Gamma(n+1)
$$

Thus, one finally can get the solution in the form:

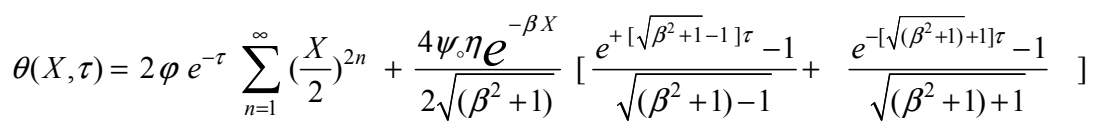

\section{Computations}

Since we are interested in the determination of the critical time required to initiate phase transition $t_{\mathrm{ph}}$ and that required to initiate melting $\mathrm{t}_{\mathrm{m}}$, we computed the obtained temperature profile $\theta(X, \tau)$ at $X=0$ for the irradiated target which is a slab of silver selenide $\left(\mathrm{Ag}_{2} \mathrm{Se}\right)$ of thickness $\mathrm{d}=0.5 \mu \mathrm{m}$.
The following laser irradiances are considered $q_{0}(t)=0.5$ $\times 10^{12} \mathrm{~W} / \mathrm{m}^{2}, 0.7 \times 10^{12} \mathrm{~W} / \mathrm{m}^{2}, 1.0 \times 10^{12} \mathrm{~W} / \mathrm{m}^{2}$. For a source of constant strength $[8,19,20]$ let us take $\eta(\tau)=1, \beta=1, \mu=$ $10^{7} \mathrm{~m}^{-1}$. Also, $\mathrm{t}_{\mathrm{k}}=1000 \mu \mathrm{s}, \mathrm{W}=0.0197 \mathrm{~m} / \mathrm{sec},(1-\mathrm{R})=0.67$ [20]. The physical, optical and thermal properties of the silver selenide slab material $[21,22]$ are given in table 1 .

Table 1. The physical, optical and thermal properties of the silver selenide slab material [21, 22].

\begin{tabular}{lllllll}
\hline & $\boldsymbol{\rho} \mathbf{k g m}^{-3}$ & $\lambda, \mathbf{W m}^{-1} \mathbf{K}^{-1}$ & $\boldsymbol{\alpha}, \mathbf{~ m}^{2} \mathbf{s e c}^{-1}$ & $\mathbf{C}_{\mathrm{p}}, \mathbf{J} \mathbf{k g}^{-1} \mathbf{K}^{-1}$ & $\mathbf{T}_{\mathbf{m}}, \mathbf{K}$ & $\mathbf{T}_{\mathrm{ph}}, \mathbf{K}$ \\
\hline silver selenide & 8200 & 1.08 & $3.9 \times 10^{-7}$ & 277 & 855 & 403 \\
\hline
\end{tabular}

The temperature of the irradiated front surface as a function of the exposure time for different indicated irradiances are given in table 2 , table 3 and table 4 respectively.

Table 2. The temperature of the front surface as function of the exposure time $q_{0}(t)=0.5 \times 10^{12} \mathrm{~W} / \mathrm{m}^{2}, d=0.5 \mu \mathrm{m}, W=0.0197 \mathrm{~m} \mathrm{sec}^{-1}, t_{k}=1000 \mu \mathrm{s}$.

\begin{tabular}{ll}
\hline $\boldsymbol{t},(\boldsymbol{\mu s})$ & $\boldsymbol{\theta ( 0 , t ) , ( \boldsymbol { K } )}$ \\
\hline 100 & 100.23 \\
120 & 143.48 \\
140 & 194.21 \\
160 & 252.3 \\
180 & 317.69 \\
200 & 390 \\
220 & 470.85 \\
240 & 556.99 \\
260 & 650.98 \\
280 & 752.02 \\
300 & 860.12 \\
\hline
\end{tabular}

Table 3. The temperature of the front surface as function of the exposure time $q_{0}(t)=0.7 \times 10^{12} \mathrm{~W} / \mathrm{m}^{2}, d=0.5 \mu \mathrm{m}, \mathrm{W}=0.0197 \mathrm{~m} \mathrm{sec}^{-1}, t_{k}=1000 \mu \mathrm{s}$.

\begin{tabular}{ll}
\hline $\boldsymbol{t},(\boldsymbol{\mu} \boldsymbol{s})$ & $\boldsymbol{\theta}(\mathbf{0}, \boldsymbol{t}),(\boldsymbol{K})$ \\
\hline 100 & 140.32 \\
120 & 200.88 \\
140 & 271.89 \\
160 & 353.22 \\
180 & 444.77 \\
200 & 546.43 \\
220 & 658.12 \\
240 & 779.78 \\
260 & 911.36 \\
\hline
\end{tabular}

Table 4. The temperature of the front surface as function of the exposure time $q_{0}(t)=1 \times 10^{12} \mathrm{~W} / \mathrm{m}^{2}, d=0.5 \mu \mathrm{m}, \mathrm{W}=0.0197 \mathrm{~m} \mathrm{sec}^{-1}, t_{k}=1000 \mu \mathrm{s}$.

\begin{tabular}{ll}
\hline $\boldsymbol{t},(\boldsymbol{\mu s})$ & $\boldsymbol{\theta}(\mathbf{0}, \boldsymbol{t}),(\boldsymbol{K})$ \\
\hline 90 & 162.86 \\
100 & 200.45 \\
\hline
\end{tabular}




\begin{tabular}{ll}
\hline $\boldsymbol{t},(\boldsymbol{\mu s})$ & $\boldsymbol{\theta ( 0 , t ) , ( \boldsymbol { K } )}$ \\
\hline 110 & 241.83 \\
120 & 286.97 \\
130 & 335.84 \\
140 & 388.41 \\
150 & 444.68 \\
160 & 504.6 \\
170 & 548.18 \\
180 & 635.38 \\
190 & 706.19 \\
200 & 780.61 \\
210 & 858.61 \\
\hline
\end{tabular}

$t_{m}$ for each value of the laser irradiance. The obtained values of the $t_{p h}$ and $t_{m}$ are tabulated in table 5 and are illustrated graphically in figure 1 as function of the laser irradiance.

Table 5. The variation of the time required to initiate phase transition $t_{p h}$ and that required to initiate melting $t_{m}$ as function of the laser irradiance $(d=0.5$ $\mu m$ ).

\begin{tabular}{lll}
\hline $\mathbf{q},\left(\mathbf{W m}^{-2}\right)$ & $\mathbf{t}_{\mathbf{p h}},(\boldsymbol{\mu s})$ & $\mathbf{T}_{\mathbf{m}},(\boldsymbol{\mu} \mathbf{s})$ \\
\hline $0.5 \times 10^{12}$ & 203.5 & 299.10 \\
$0.7 \times 10^{12}$ & 171.3 & 251.63 \\
$1 \times 10^{12}$ & 142.7 & 209.56 \\
\hline
\end{tabular}

Form the corresponding data, one can determine both $\mathrm{t}_{\mathrm{ph}}$ and

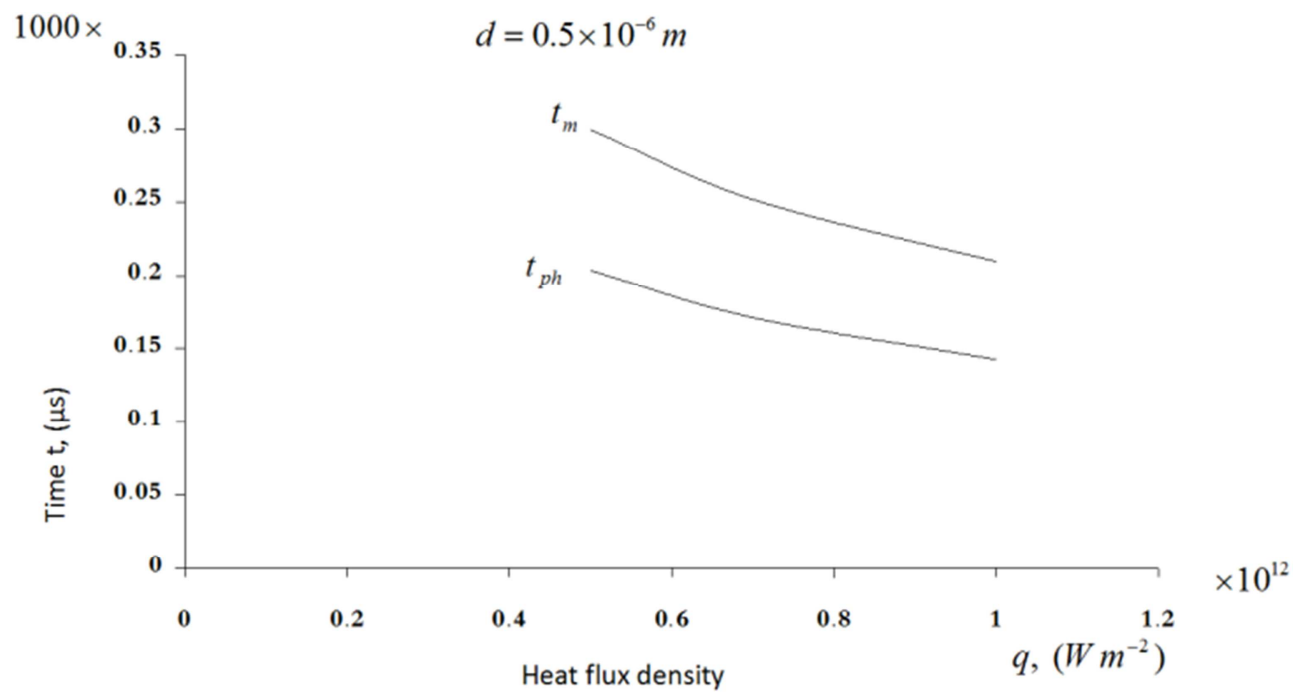

Figure 1. The dependence of $t_{p h}$ and $t_{m}$, on the heat flux density $\left(\mathrm{W} / \mathrm{m}^{2}\right)(d=0.5 \mu \mathrm{m})$.

\section{Conclusion}

As a result of the obtained temperature profiles and computed data the following conclusions can be summarized as follows:

1. The temperature of the front surface of the irradiated target does depend linearly on the maximum value of the received laser irradiance. That is not the case for the profile within the whole target.

2. The critical time required to initiate phase transition and that required to initiate melting at the front surface have nonlinear dependence on the thickness of the irradiated slab. Such dependence is controlled through the function $\exp (-\beta x)$ defined in the text.

3. The obtained mathematical expressions for the temperature profile specify the optimum conditions to realize either phase transition only or melting or both.

In general the obtained results in this study have vital importance in the field of technological applications for laser interaction with solid matter.

\section{References}

[1] Ready J. F., Effects due to absorption of laser radiation, J. Appl. Phys., Vol. 36 pp. $462-468$ (1965).
[2] Ready J. F., Industrial Application of Lasers - New York, Academic Press (1978).

[3] Brorson S. D., Fujinoto J. G. Jappen E. P., Femtosecond electronic heat- transport dynamics in thin gpld films, Phys. Rev. lett. 59: 1962-1965 (1978).

[4] El- Adawi, M. K., Laser melting of solids- An exact solution for time intervals less or equal to the transit time, J. Appl. Phys. vol. 60 No. (7) pp. 2256-2259 (1986).

[5] El-Adawi M. K., E. F. El-shehawey, Heating a slab induced by a time dependent laser irradiance-An exact Solution, J. Appl. Phys. Vol. 60 No. (7) pp. 2250-2255 (1986).

[6] Olstad R. A., Olander D. R., Evaporation of solids by laser pulses, J. Appl. Phys. vol. 46, pp. 1499-1508 (1975).

[7] Porteus J. O, Choyke W. T. and Hoffman R. A., Pulsed Laser damage characteristic of vapor-deposited copper mirrors on Silicon Carbide substrate, Appl. Opt. vol 9, pp., 451-454 (1980).

[8] Lewandowska M., Hyperbolic heat conduction in the semiinfinite body with a time- dependent laser heat source, Heat and Mass Transfer, Vol. 37, pp. 333-342 (2001).

[9] Miki M., super formation and phase transition in silver selenide, J. Appl. Phys. vol. 30, No. 8, pp. 1765-1769 (1991).

[10] Kumar MC. S, Pradeep B., structural, electical and optical properties of silver selenide thin films, Semiconductor Science and Technology vol. 7, pp. 261-265 (2002). 
[11] Schoen, D. T., Yie C., Cui Y., Electerical switching and phase transformation in silve selenide nanowires, J. of the American Chemical Society vol. 129, No. 14, pp. 4116-.(2007) 4117

[12] Zubair SM., Aslam Chaudhry M., Heat conduction in a semiinfinite solid due to time-dependent laser source., Int. J. Heat Mass Transfer vol. 39 pp. 3069-3074 (1996).

[13] Ghez R. A. and Laff. R. A., Laser heating and melting of thin films in low- conductivity substrate, J. Appl Phys., Vol. 46 (5) pp. 2103-2110 (1975).

[14] El- Adawi M. K., Shalaby S. A., Pulsed Laser heating of a finite silve selenide slab using (HHCE) model, Applied Mathematics, vol. 9 pp..355-368 (2018).

[15] Rainville E. D., Bedient P. E., Elementary Differential equations $5^{\text {th }}$ edition, Macmillan Publishing Co. New York, Ch. 9 pp. 144 (1974).

[16] Roberts G. E and Kaufman H., Tables of Laplace Transforms. Saunders Company Lando, pp. 254 (1980).
[17] Gradshteyn I. S., Ryzhik I. M., Table of integrals, series, and products, Academic Press, New York (1980).

[18] Murray R. Spiegel. Schaum's Outline Series, Laplace Transforms. McGraw-Hill Book Company, New York (1880).

[19] Hector L. G., Kim W. S., Ozisik M. N., Propagation and reflection of thermal waves in a finite medium due to axisymmetric surface sources., Int J. Heat Mass Trans. 35: 897-912 (1992).

[20] Bhattacharyya A., and. Streetman B. G., Dynamics of pulsed laser annealing of silicon, J. Phys. D: Appl Phys., 14: L 67-72 (1981).

[21] Dean J. A., Lange's handbook of chemistry. McGraw-Hill Book Company, New York, pp. 126-127 (1978).

[22] West A. R., Basic solid state chemistry, John Wiley \& Sons (1999). 